\title{
Planning and Simulation of Robotically Assisted Minimal Invasive Surgery ${ }^{\star}$
}

\author{
Louaï Adhami, Ève Coste-Manière, and Jean-Daniel Boissonnat \\ INRIA Sophia-Antipolis B.P. 93 FR-06902 Sophia-Antipolis, France. \\ \{Louai.Adhami, Eve.Coste-Maniere, Jean-Daniel.Boisonnat\}@sophia.inria.fr \\ http://www.inria.fr/chir
}

\begin{abstract}
This paper proposes a framework for pre-operative planning and simulation of robotically assisted Minimal Invasive Surgery (MIS). The design of an integrated system is presented for cardiovascular interventions. The approach consists of a planning, validation and simulation phase. The goals of each phase being, respectively, to propose suitable incision sites for the robot, to validate those site and to enable realistic simulation of the intervention. With the patient's pre-operative data, we formulate the needs of the surgeon and the characteristics of the robot as mathematical criteria in order to optimize the settings of the intervention. Then we automatically reproduce expected surgeons' movements and guaranty their feasibility. Finally we simulate the intervention in real-time, paying particular attention to potential collisions between the robotic arms.
\end{abstract}

Keywords: Surgical simulators, robotics and robotic manipulators, therapy planning.

\section{Introduction}

The extension of MIS techniques to use robotics came from the limitations imposed on the surgeon by manually controlled MIS instruments. Namely, the surgeon finds his movements, vision and tactile sensing reduced and severely altered $(9], 3])$. The introduction of robotic manipulators would remedy the loss of dexterity in the movements by incorporating additional degrees of freedom at the end of the tools; i.e., in the part that would be inside the patient. In addition, more precision and better tactile feedback can be achieved by proper design of the system, which can also improve the hand eye alignment of the surgeon and offer better $3 \mathrm{~d}$ visualization.

However, this innovation has its own limitations and problems. Beginning with the limitations, and despite the increased dexterity, the region reached from a set of incision sites will remain restrained, thus these sites have to be carefully chosen for each patient depending on his anatomy. Moreover, the forces that can be delivered by a robotic manipulator may vary significantly with the position

\footnotetext{
* This work is partially supported by the Télémédecine project of the French Ministry of Research.
} 
of the latter, which stresses even more on the choice of the incision sites or ports. Now moving to the problems introduced by the use of a robot, and setting aside classical control and liability concerns, the main handicap of such systems is the issue of potential collisions between the manipulator arms. Again this stresses on the proper positioning of both the incision ports and the robot. Finally, and no matter how intuitive the controlling device is made, the surgeon will need time and proper training before using his new "hands" in the most efficient way. Therefore simulation would be used to rehearse the intervention, validating the planned ports and helping the surgeon gets accustomed to both his tools and his patient.

In short, a strong need of planning has been pointed out for robotically assisted MIS, as well as suitable simulation. These, along with necessary intermediate steps will be described in this paper as a generic methodology for robotically assisted MIS. However, and in order to have an illustrative example, the MIDCAB (minimally invasive direct coronary artery bypass) intervention was chosen as a test bed for our work. The MIDCAB intervention is a closed chest cardiac intervention where damaged portions of cornary vessels are bypassed. This choice is based on the importance of the MIBCAD intervention, and on the sub-millimeter precision needed to perform it, which makes the use of a robotic manipulator very attractive. Likewise, a prototype version of the daVinci ${ }^{\mathrm{TM}}$ robot 11 was selected for the implementation of our approach.

The proposed work is part of a more complete effort of computer assistance for MIS interventions which comprises, in addition to the pre-operative phase presented here, an augmented reality intra-operative guidance system. Thus during the intervention, the surgeon will be guided in accordance with his pre-operative plans, and online validation will insure a safe, collision free manipulation of the robot.

Related Work The use of computer assisted systems in the preparation and simulation of minimally invasive techniques is being recognized as an efficient tool in surgery. Various successful systems have been proposed and are already operational. Some examples are the haptic system based on the work of [4, the more general purpose system in 7 , and 2 in neurosurgery. These systems do not handle teleoperated robotics, with the notable exception of the KISMET simulation tool that is being merged with the ARTEMIS robotic environment (see [1]), but which lacks systematic planning.

In the case of robotically assisted MIDCAB intervention, existing planning attempts are rather empirical (9] and [10]). A more systematic effort is found in [6], where pre-operative data is used to measure the distance between the concerned vessels. However, no robotic system is used, and a mini-thoracotomy is performed. To the best of our knowledge, there presently is no system that integrates the planning, simulation and online control of a robotic surgery system for laparoscopic or cardiac MIS interventions.

\footnotetext{
${ }^{1}$ The da Vinci surgical system is a trademark of Intuitive Surgical Inc.
} 


\section{Overview}

Our approach, schematically summarized in figure 1, can be subdivided in four main parts outlined below.

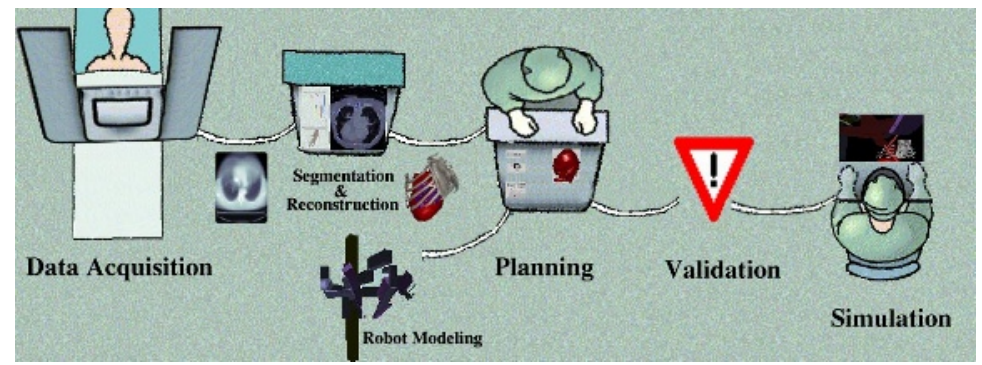

Fig. 1. General Approach.

1. Preliminary processing: Pre-operative radiological data is often difficult to handle; therefore, suitable pre-processing such as segmentation and $3 \mathrm{~d}$ reconstruction should first be carried out. Since the data used is patient dependent, it is also desirable to have a completely automatic process to handle the output of a radiological imaging device directly. Moreover the robot system that will be used has to be modeled.

2. Planning: The goal of the planning phase is to determine the best incision sites for the introduction of the robot arms and endoscope. For that, three sources of information are combined in an optimization algorithm where some predefined mathematical criteria have been integrated. The criteria translate features such as collision avoidance between the manipulator arms or reachability of targeted organs.

3. Validation: Once a set of ports is obtained, the feasibility of the intervention is checked by automatically reproducing the expected movements of the surgeon and looking for collisions or other problems, such as an out of reach condition.

4. Simulation: Finally, the surgeon is presented with the incision ports and he is now ready to test them, and may require another set if he judges the proposed one not satisfactory. In addition, he would be able to "rehearse" his intervention, thus comparing different strategies and getting accustomed to the use of the robot.

\section{Preliminary Steps}

Radiological data must first be processed and combined with the robot model in order to carry on with the planning. A set of anatomical entities has to be 
isolated for every type of surgical intervention. Moreover, some special handling may be required for the port placement to allow or forbid access to certain physiological parts.

\subsection{Segmentation}

The patients' anatomical entities that are of interest are isolated using a novel knowledge based segmentation technique that will be the subject of a future publication. The process is invariant from one patient to another, in particular it does not have any parameters to be tuned by the radiologist. However, it should be noted that manual interventions are still required at this stage.

For all robotically assisted MIS intervention, we define the admissible points as those sites that allow the introduction of the robot arms and endoscope. They are coupled with admissible directions pointing outward and perpendicular to the skin, which will be used during the planning phase. Figure 2 shows a segmentation for the MIDCAB intervention, where the admissible sites are the intercostal spaces. Additional algorithms were added to compute the density of the traversed ray from the admissible point to the skin in the normal direction, in order to determine whether a proposed port is acceptable or not; e.g, a ray passing through a bony structure is eliminated.
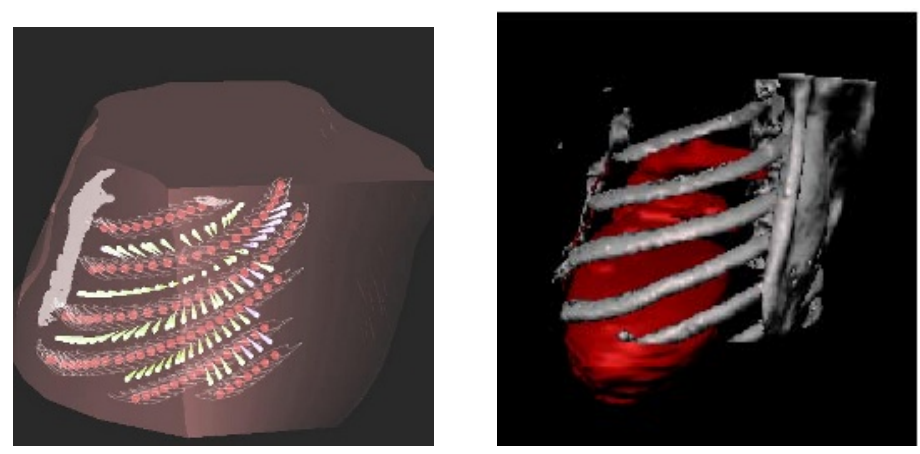

Fig. 2. (a) The admissible ports and their directions, the rib centers are shown as spheres. (b) Using nuages to reconstruct in $3 \mathrm{~d}$ the anatomical entities needed for the MIDCAB intervention.

\subsection{Reconstruction}

$3 \mathrm{~d}$ reconstruction is essential for efficient visualization and manipulation of the segmented data. The software used for the reconstruction is called nuages and is described in [5]. The underlying algorithm is based on projected Voronoï diagrams, where the input is a set of closed non-intersecting contours, and the output is a mesh of triangles representing the reconstructed surface in $3 \mathrm{~d}$. This 
algorithm has the advantages of outputting a low and controllable number of triangles, and of not being prone to distortive effects such as the staircase effect observed in marching cubes algorithms. On the other hand, its input is somewhat restrictive, as only non-intersecting slice-wise closed curves are allowed. Figure 2 (b) depicts a reconstruction for the MIDCAB intervention.

\subsection{Robot Modeling}

Modeling the robot is an essential step that will gain in importance as we advance through the stages of our approach. In the planning phase, only the DenavitHartenberg (DH) models are needed. For validation, an implementation of the kinematics have to be carried out, and finally the simulation would incorporate as much as possible the dynamics of the system.

To carry out the modeling, we developed a generic $\mathrm{C}++$ library, where OpenGL ${ }^{R}$ output and collision detection are implemented. Two primitives were retained for the modeling of the robot body, namely rectangular parallelepipeds and cylinders. Moreover, inverse kinematics are carried out either analytically or numerically. An analytic solution is used when there is the same number of degrees of freedom (dofs) and constraints, whereas a numerical solution is used when there are more dofs than constraints. In the latter case, artificial constraints are added to reflect the proximity between the arms, which would be of great significance when dealing with the problem of collision avoidance.

\section{Planning}

As already mentioned, the choice of the incision points for the robot arms and endoscope are critical to the success of the intervention. Therefore, some sort of optimization is desired in order to guarantee the best possible locations of the ports, if such positions exist. Naturally, the best way to insure the feasibility of the intervention is through simulation, which is the object of section 6 Nevertheless, one cannot try out all possible configurations, even if driven by some experience and common sense. The proposed solution is to choose from the set of all possible candidates, the admissible points, a triplet that optimizes a set of predefined criteria. These criteria are based on the descriptions of the surgeons' difficulties and priorities when performing MIS interventions, whether robotically assisted or not.

\subsection{Criteria}

The first step is for the surgeon to specify his points of interest, or target points, along with desired attack direction and area (see figure 4 (b) for an example). The target points are representatives of the areas on which the surgeon wants to operate, whereas the attack direction specifies the orientation of the surgical tool with respect to the surface at the attack point. This defines the criteria considered in the optimization process, which are: 
1. The target area, which is the area chosen by the surgeon to translate the importance of the site on which he wishes to work.

2. The attack angle, which is the angle between the attack direction at the target point on the one hand, and the straight line connecting the latter to the admissible point on the other. It reflects the ease with which the surgeon can operate on a given location with respect to the ideal inclination he had specified.

3. The dexterity parameter, which is proportional to the angle between the surface normal at the admissible point and the straight line connecting the latter to the target point. This measure of dexterity has to be interpreted in accordance with the robot capabilities.

\subsection{Optimization}

In order to isolate the most interesting triplet according to the above enumerated criteria, the following steps are carried out.

1. Eliminate admissible candidates that are not fully accessible from the ports by rendering a cone with its apex at the admissible point and its base covering the target area (see figure $3(\mathrm{a})$ ). The technique of rendering parts of scene to detect interference was inspired from 8 .

2. For the endoscope, sort the admissible sites in a way to minimize the angle between the target normal and the line connecting the admissible point to the target point. This step privileges a direct view over the areas of interest. It should also be noted that the targets are weighted in accordance to their area. Moreover points with angles greater than the camera angle used during the intervention, are eliminated.

3. For the arms, sort in the same way as for the endoscope, but with the angle limitation relaxed. Moreover, eliminate admissible candidates that make to obtuse an angle this time with the normal to the skin. For example $60^{\circ}$ is the limit in the MIDCAB case to avoid excessive stress on the ribs. The different angles are shown in figure 3 (b). Moreover, a check is performed on the different dimensions of the links to insure that no movement will cause an out of reach condition.

4. Finally choose the triplet that compromises the score of each site according to the above criteria with maximizing the distance between the ports. The latter requirement is a natural remedy against the problem of collision between the robot arms.

\subsection{Results}

The results obtained on a test case for the MIDCAB intervention are shown in figure 4 (b), next to those recommended by [9] in (a). The optimization is exhaustive, although hierarchical elimination is carried out to speed up the process. The evaluation of the results is ultimately through clinical trials; however, 

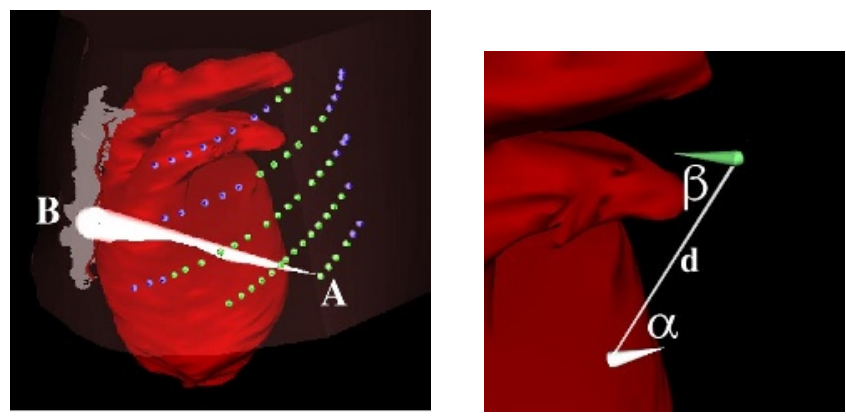

Fig. 3. (a) An example of a partially occluded area. Imagine having a flash light at point $\mathrm{A}$, the targeted area B is not fully illuminated. (b) The measures used for optimization: $\alpha$ is the angle introduced in the $2^{\text {nd }}$ step, $\beta$ in the $3^{\text {rd }}$ and $d$ the distance in $4^{\text {th }}$ step.

a first estimation will be through virtual validation and simulation discussed in section 5 and 6 These evaluations were very positive: typically one of the first three triplets passed the validation test that we now present.
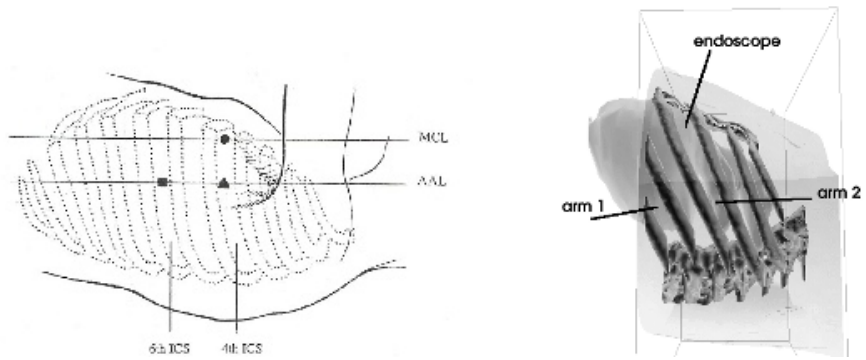

Fig. 4. Port placement results for the MIDCAB intervention: (a) Surgeons' recommendations (reproduced from [9]), and (b) optimized ports. Circle/yellow for camera port, square/triangle/cyan for tools ports.

\section{Validation}

Once a suitable triplet has been identified, the robot is placed in the proposed position (see section 3.3 for the way inverse kinematics are carried out). In order to guarantee the feasibility of the required surgical act, the current configuration is tested for a collision free path connecting the different target area. The details of interference detection are presented in section 6.1 . 
The trajectory generated between two target areas is a straight line, as this is the way the surgeon is expected to navigate. The possibility of collisions between discrete time steps are handled by the interference detection algorithm that tests sweeps the volume covered by the manipulator arms. In addition to interference detection, out of reach conditions and possible singularities are monitored and signaled. Finally, the endoscope is positioned relative to the tips of the tool arms at a predefined distance, in a way to guarantee a good visibility at all times. Figure 5 depicts a test for the MIDCAB intervention.

If no problem is detected, then the triplet is accepted and the surgeon can proceed with manual verification, else the triplet is refused and another one is computed. If no suitable ports can be found, then the robot is placed in a predefined nominal position and the surgeon would try to find one manually.
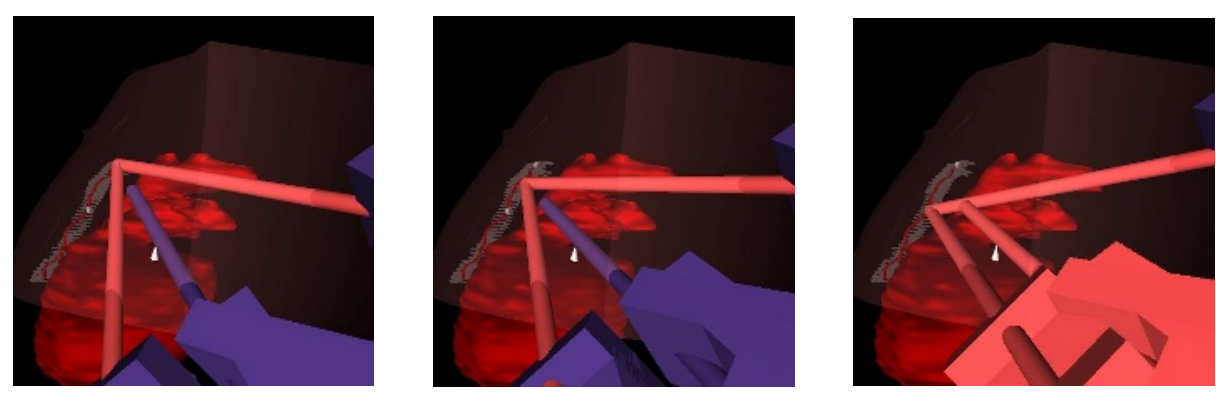

Fig. 5. A validation sequence. Note the colliding state and the out of reach condition in the last image.

\section{Simulation}

The simulator has the double aim of offering the surgeon a realistic environment to develop good control over the robot, and of validating the suggested incision ports.

\subsection{Collision Detection}

Exact real-time collision detection is an essential requirement for a realistic simulation and a safe validation of the strategy that will be used in the intervention. Collisions are stratified as external (with the anatomical entities) and internal (between the manipulators), the latter being further subdivided into static and dynamic (continuous movement). For the latter types, an algorithm to detect interferences between rectangular parallelepipeds and cylinders was written. It is capable of handling both static and dynamic collisions, thus considerably reducing the sensitivity to the discretization step. On the other hand, external collisions are detected using the method suggested in [8], which makes use of the graphics hardware. Both methods run at frame rate for typical data. 


\subsection{The Interface}

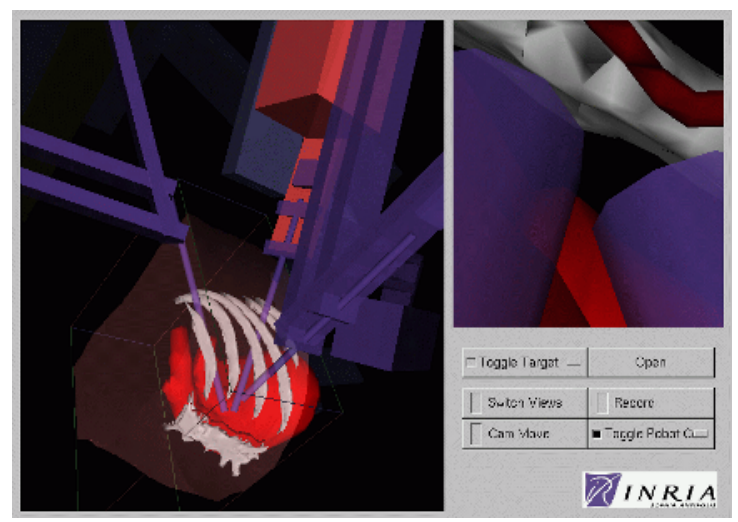

Fig. 6. A single interface is used for both planning and simulation.

Figure 6 shows a snapshot of the interface developed for surgeon interaction. Besides the usual 3d navigation and all the functionalities included for the planning phase, a realistic control of the robot has been implemented using two $3 \mathrm{~d}$ pointing device. 2 . The models used are those of the prototype of the daVinci ${ }^{\mathrm{TM}}$ system. As is the case with the actual robot, the surgeon manipulates the tools simultaneously in the camera coordinate frame, and may also control the camera using a special switch. Automatic validation may be replayed, and a new optimization may be requested at any time.

\section{Conclusion and Future Trends}

A framework for pre-operative planning and simulation of robotically assisted MIS intervention has been proposed. The three main steps are planning, validation and simulation. The results obtained in the planning phase are very encouraging and seem coherent with those proposed manually. Moreover, the interface was well accepted by the surgeons.

The validation step forms a solid guaranty of the results proposed in the planning. Efficient collision detection methods make it possible to try out different setups in a few seconds. Finally the simulation interface offers an intuitive tool to rehearse the intervention and comfort the surgeon. Current efforts are directed towards the following extensions of the system:

1. Merging the planning step with the validation step so as to directly generate the desired set of ports.

\footnotetext{
${ }^{2}$ Magellan Space Mouse, http://www.logicad3d.com.
} 
2. The integration of the present work with the intra-operative part to enable clinical validation of the results on the MIDCAB and other interventions.

3. More realistic simulation by the use of deformable models to represent soft tissues.

4. Improvement of the segmentation technique until no manual intervention is required.

5. More realistic representation of the organs; e.g, integrating a coronary map on the heart.

\section{Acknowledgements}

This work would not have been possible without the help of Prof. Alain Carpentier and Dr. Didier Loulmet from the Hôpital Broussais in Paris. We would also like to acknowledge the Intuitive Surgical Inc. team, especially Dr. Kenneth Salisbury and Dr. Gary Guthart.

\section{References}

[1] ARTEMIS homepage. http://wwwserv2.iai.fzk.de/artemis/.

[2] L. Auer, A. Radetzky, C. Wimmer, G. Kleinszig, H. Delingette, and B. Davies. Visualisation for planning and simulation of minimally invasive procedures. Lecture Notes in Computer Science, 1679:1199-1209, Sept. 1999.

[3] G. B. Cadière and J. Leroy. Principes généraux de la chirurgie laparoscopique. Encycl Méd Chir (Techniques chirurgicales - Appareil digestif), volume 40, page 9. Elsevier-Paris, 1999.

[4] S. Cotin, H. Delingette, and N. Ayache. Real-time elastic deformations of soft tissues for surgery simulation. IEEE Transactions On Visualization and Computer Graphics, 5(1):62, 1999.

[5] B. Geiger. Three dimentional modeling of human organs and its application to diagnosis and surgical planning. Technical Report 2105, INRIA-Sophia, 1993.

[6] H. Gulbins, H. Reichenspurner, C. Bechker, D. Boehm, A. Knez, C. Schmitz, R. Bruening, R. Harbrel, and B. Reichart. Preoperarive 3d-reconstruction of the ultrafast-CT images for the planning of the minimally invasive direct coronary artery bypass operation (MIDCAB). In Second World Congress of Minimally Invasive Cardiac Surgery, Minneapolis, Minnesota, June 1998.

[7] U. Kühnapfel, H. Çakmak, and H. Maaß. 3Dmodeling for endoscopic surgery. In Proc. IEEE Symposium on Simulation, pages 22-32, Delft University, Delft, NL, Oct. 1999.

[8] J.-C. Lombardo, M.-P. Cani, and F. Neyret. Real-time collision detection for virtual surgery. In Computer Animation, Geneva, May 1999.

[9] D. Loulmet, A. Carpentier, N. d'Attellis, A. Berrebi, C. Cardon, O. Ponzio, B. Aupècle, and J. Y. M. Relland. Endoscopic coronary artery bypass grafting with the aid of robotic assisted instruments. The journal of thoraic and cardiovascular surgery, 118(1), July 1999.

[10] H. Tabaie, J. Reinbolt, P. Graper, T. Kelly, and M. Connor. Endoscopic coronary artery bypass graft (ECABG) procedure with robotic assistance. The Heart Surgery Forum (http://www.hsforum.com), 2(0552), Sept. 1999. 